\title{
On Transmit Beamforming for Multiantenna OFDM Channels With Finite-Rate Feedback
}

\author{
Kritsada Mamat and Wiroonsak Santipach \\ Department of Electrical Engineering \\ Faculty of Engineering, Kasetsart University \\ Bangkok, Thailand 10900 \\ Email: $\{$ g5317500192, wiroonsak.s\}@ku.ac.th
}

\begin{abstract}
We propose two feedback methods for transmit beamforming in a point-to-point multiple-antenna OFDM channel. For the first method, a receiver with channel information quantizes and feeds back the optimal transmit beamforming vectors of a few selected subcarriers that are equally spaced. Based on those quantized vectors, the transmitter linearly interpolates the remaining beamforming vectors with different phase rotation whose expression is explicitly shown. For the second proposed method, a channel impulse response is quantized with a uniform scalar quantizer. At the transmitter, channel frequency response can be reconstructed from the quantized impulse response and the optimal beamforming vectors can then be computed. We show that switching between the two methods for different feedbackrate requirement outperforms existing methods in the literature.
\end{abstract}

\section{INTRODUCTION}

Equipping a transmitter and a receiver with multiple antennas creates multiple-input multiple-output (MIMO) wireless channel whose capacity depends on channel information available at the transmitter and receiver. In multiantenna channel, transmit beamforming has been shown to increase channel capacity by directing transmit signal toward the strongest channel mode [1]. With channel information, the receiver can compute the optimal beamforming vector that maximizes channel capacity and feeds the vector back to the transmitter. Due to a finite feedback rate, the beamforming vector needs to be quantized. Several quantization schemes and codebooks have been proposed and analyzed, and the corresponding performance was shown to depend on a codebook design and the number of available feedback bits [2], [3, see references therein].

In this work, we consider transmit beamforming for multiple-antenna orthogonal frequency-division multiplexing (OFDM), which converts a wideband channel into parallel narrowband subchannels. For each subchannel or subcarrier, the optimal beamforming vector is different and needs to be quantized and fed back. The total number of feedback bits required increases with the number of subcarriers, which can be large. Reference [4]-[7] have proposed to reduce feedback amount while maintaining a performance. In [5], the optimal

This work was supported by the 2010 Telecommunications Research and Industrial Development Institute (TRIDI) scholarship and a joint funding from Thailand Commission on Higher Education, Thailand Research Fund, and Kasetsart University under grant MRG5580236. transmit beamforming vectors of selected subcarriers, which are a few subcarriers apart, is quantized while the rest is approximated to equal the quantized vector of the closest subcarrier. In [4] and [6], the rest of transmit beamforming vectors are proposed to be linearly interpolated and spherically interpolated, respectively. In [7], a channel impulse response is vector quantized and fed back to the transmitter where the frequency response can be reconstructed.

For a low feedback rate, we propose to quantize the optimal beamforming vector at every few subcarriers with random vector quantization (RVQ) codebook proposed by [2] and to linearly interpolate the remaining beamforming vectors from the quantized vectors. The proposed interpolation method is improved upon the work by [4] by deriving a closed-form expression for the phase-rotation parameter instead of having to perform exhaustive search to locate the parameter. The derived phase rotation is mainly a function of correlation between adjacent subcarriers. When a feedback rate is extremely limited, the proposed method is shown to outperform the interpolation by [4] for both multiple-input single-output (MISO) and MIMO channels. Numerical results show that for a given feedback rate and a number of fading paths, there exists the optimal subcarrier interval that maximizes the capacity.

When the feedback rate is high, we propose to quantize the channel impulse response with a uniform scalar quantizer and derive the approximate capacity upper bound for MISO channel. A scalar quantization used in the proposed method is less complex than a vector quantization used in [7]. The proposed scalar quantization of channel impulse response is shown to perform well for MISO channel. Similar results are observed by [2] where the optimal beamformer and not channel response is scalar quantized.

\section{System MODEL}

We consider a point-to-point OFDM channel with $N_{t}$ transmit and $N_{r}$ receive antennas, and $N$ subcarriers. Antenna arrays at both transmitter and receiver are assumed to be sufficiently large that all antenna pairs are independent. For each transmit-receive antenna pair, a transmitted signal propagates through a frequency-selective Rayleigh fading channel with order $L$. We denote a discrete-time channel impulse response between the $n_{t}$ th transmit and the $n_{r}$ th receive antennas by 
an $L \times 1$ vector $\boldsymbol{h}_{n_{r}, n_{t}}=\left[\begin{array}{llll}h_{n_{r}, n_{t}}^{(0)} & h_{n_{r}, n_{t}}^{(1)} \cdots & h_{n_{r}, n_{t}}^{(L-1)}\end{array}\right]^{T}$. Assuming a uniform power delay profile for all antenna pairs, each channel tap $h_{n_{r}, n_{t}}^{(l)}$ is an independent complex Gaussian random variable with zero mean and variance $\frac{1}{L}$.

A frequency response at the $n$th subcarrier for the $\left(n_{r}, n_{t}\right)$ antenna pair is given by a discrete Fourier transform (DFT) of the $L$-tap impulse response as follows

$$
H_{n_{r}, n_{t}}(n)=\boldsymbol{h}_{n_{r}, n_{t}}^{T} \boldsymbol{D}_{n}=\sum_{l=0}^{L-1} h_{n_{r}, n_{t}}^{(l)} \mathrm{e}^{-\frac{j 2 \pi l n}{N}}
$$

where $\boldsymbol{D}_{n}=\left[\begin{array}{lllll}1 & \mathrm{e}^{-\frac{j 2 \pi n}{N}} & \mathrm{e}^{-\frac{j 2 \pi 2 n}{N}} & \cdots & \mathrm{e}^{-\frac{j 2 \pi(L-1) n}{N}}\end{array}\right]^{T}$ and the subscript $T$ denotes matrix transpose. Let $\boldsymbol{H}_{n}$ denote an $N_{r} \times N_{t}$ channel matrix for the $n$th subcarrier, whose entry is $H_{n_{r}, n_{t}}(n)$ shown in (1).

Applying a transmit beamforming or a rank-one precoding, the $N_{r} \times 1$ received vector on the $n$th subcarrier is given by

$$
\boldsymbol{r}_{n}=\boldsymbol{H}_{n} \boldsymbol{v}_{n} x_{n}+\boldsymbol{z}_{n} \quad \text { for } 0 \leq n \leq N-1
$$

where $\boldsymbol{v}_{n}$ is an $N_{t} \times 1$ unit-norm beamforming vector, $x_{n}$ is a transmitted symbol with zero mean and unit variance, $\boldsymbol{z}_{n}$ is an $N_{r} \times 1$ AWGN vector with zero mean and covariance $\sigma_{z}^{2} \boldsymbol{I}$, and $\boldsymbol{I}$ is an identity matrix. We assume a uniform power allocation in which each subcarrier is allocated equal transmit power. Hence, the background signal-to-noise ratio (SNR) for each subcarrier $\rho=1 / \sigma_{z}^{2}$. The corresponding sum capacity over all subcarriers is given by

$$
C=\sum_{n=0}^{N-1} E_{\boldsymbol{H}_{n}}\left[\log \left(1+\rho \boldsymbol{v}_{n}^{\dagger} \boldsymbol{H}_{n}^{\dagger} \boldsymbol{H}_{n} \boldsymbol{v}_{n}\right)\right]
$$

where the expectation is over a distribution of $\boldsymbol{H}_{n}$. From (3), we note that the sum capacity is a function of transmit beamforming vectors of all subcarriers, $\left\{\boldsymbol{v}_{0}, \boldsymbol{v}_{1}, \ldots, \boldsymbol{v}_{N-1}\right\}$.

\section{INTERPOLATING TRANSMIT BEAMFORMING VECTORS}

Feeding back transmit beamforming vectors of all subcarriers requires quantizing $N N_{t}$ complex coefficients. To curb quantization error, a large number of feedback bits is needed. We note that adjacent subcarriers in OFDM are highly correlated since the number of channel taps is much lower than that of subcarriers $(L \ll N)$. Hence, the optimal transmit beamformers, which depend on channel matrices, are also highly correlated as well.

To reduce the number of feedback bits, we propose to only quantize a optimal transmit beamformer of every $M$ subcarriers and to interpolate the rest of the transmit beamformers. In this work, RVQ [2] is used to quantize the beamforming vector. An RVQ codebook consists of independent isotropically distributed vectors and was shown to perform close to the optimum codebook [2], [8]. Let $K \triangleq\lfloor N / M\rfloor$ denote the number of quantized beamformers and let $B$ denote the total number of available feedback bits for each feedback update from the receiver. Thus, the number of bits used to quantize each optimal beamforming vector is $B / K$. With RVQ codebook denoted by $\mathcal{V}=\left\{\boldsymbol{w}_{1}, \boldsymbol{w}_{2}, \ldots, \boldsymbol{w}_{2^{B / K}}\right\}$, the receiver selects the transmit beamforming vector for the $k M$ th subcarrier that maximizes the achievable rate as follows

$$
\begin{aligned}
\boldsymbol{v}_{k M} & =\arg \max _{\boldsymbol{w} \in \mathcal{V}} \log \left(1+\rho \boldsymbol{w}^{\dagger} \boldsymbol{H}_{k M}^{\dagger} \boldsymbol{H}_{k M} \boldsymbol{w}\right) \\
& =\arg \max _{\boldsymbol{w} \in \mathcal{V}} \boldsymbol{w}^{\dagger} \boldsymbol{H}_{k M}^{\dagger} \boldsymbol{H}_{k M} \boldsymbol{w}
\end{aligned}
$$

where $0 \leq k \leq K-1$.

Between $k M$ th and $(k+1) M$ th subcarriers, the unit-norm beamforming vector is interpolated as follows [4]

$$
\boldsymbol{v}_{k M+m} \triangleq \frac{\left(1-c_{m}\right) \boldsymbol{v}_{k M}+c_{m} e^{j \theta_{m}} \boldsymbol{v}_{(k+1) M}}{\left\|\left(1-c_{m}\right) \boldsymbol{v}_{k M}+c_{m} e^{j \theta_{m}} \boldsymbol{v}_{(k+1) M}\right\|}
$$

for $1 \leq m \leq M-1$, where $c_{m}=\frac{m}{M}$ is a linear weight and $\theta_{m}$ is a phase-rotation parameter. In [4], $\theta_{m}$ is chosen to maximize the sum capacity in (3) by performing exhaustive search over the received power. To avoid search complexity, we propose to determine the phase rotation based on a correlation between the optimal beamformers of neighboring subcarriers.

\section{A. MISO Channel}

We note that for MISO channel $\left(N_{r}=1\right)$, the optimal transmit beamforming vector for the $n$th subcarrier is the normalized channel vector

$$
\boldsymbol{v}_{n}^{\text {opt }}=\boldsymbol{H}_{n}^{\dagger} /\left\|\boldsymbol{H}_{n}\right\|
$$

Based on numerical simulations, a correlation between optimal beamforming vectors that are $q$ subcarriers apart, can be approximated as follows

$$
\begin{aligned}
E\left|\left(\boldsymbol{v}_{n}^{\mathrm{opt}}\right)^{\dagger} \boldsymbol{v}_{n+q}^{\mathrm{opt}}\right|^{2} & =E\left[\frac{\left|\boldsymbol{H}_{n} \boldsymbol{H}_{n+q}^{\dagger}\right|^{2}}{\left\|\boldsymbol{H}_{n}\right\|^{2}\left\|\boldsymbol{H}_{n+q}\right\|^{2}}\right] \\
& \approx \frac{E\left|\boldsymbol{H}_{n} \boldsymbol{H}_{n+q}^{\dagger}\right|^{2}}{E\left[\left\|\boldsymbol{H}_{n}\right\|^{2}\left\|\boldsymbol{H}_{n+q}\right\|^{2}\right]} .
\end{aligned}
$$

With some algebraic manipulation and the fact that all the channel taps are independent Gaussian distributed, we can show that for $N_{r}=1$,

$$
E\left|\boldsymbol{H}_{n} \boldsymbol{H}_{n+q}^{\dagger}\right|^{2}=N_{t}+\frac{N_{t}^{2}}{L^{2}} \varphi^{2}(q)
$$

and

$$
E\left[\left\|\boldsymbol{H}_{n}\right\|^{2}\left\|\boldsymbol{H}_{n+q}\right\|^{2}\right]=N_{t}^{2}+\frac{N_{t}}{L^{2}} \varphi^{2}(q)
$$

where $\varphi(x) \triangleq \frac{\sin \left(\frac{\pi x L}{N}\right)}{\sin \left(\frac{\pi x}{N}\right)}$. Substituting (10) and (11) in (9), we obtain

$$
E\left|\left(\boldsymbol{v}_{k M}^{\mathrm{opt}}\right)^{\dagger} \boldsymbol{v}_{k M+m}^{\mathrm{opt}}\right|^{2} \approx \frac{L^{2}+N_{t} \varphi^{2}(m)}{L^{2} N_{t}+\varphi^{2}(m)} \triangleq \psi\left(m, N_{t}\right) .
$$

Evaluating a correlation between the optimal beamformer and the interpolated beamformer that are $m$ subcarriers apart, $E\left|\left(\boldsymbol{v}_{k M}^{\text {opt }}\right)^{\dagger} \boldsymbol{v}_{k M+m}\right|^{2}$, follows similar steps. We conjecture that this correlation equals the correlation between the optimal beamformers, which is approximated to be $\psi\left(m, N_{t}\right)$ in (12). Based on the conjecture, we set $E\left|\left(\boldsymbol{v}_{k M}^{\text {opt }}\right)^{\dagger} \boldsymbol{v}_{k M+m}\right|^{2}$ to $\psi\left(m, N_{t}\right)$ and solve for the phase-rotation parameter given by

$$
\theta_{m}=\arccos \frac{U(m)}{V(m)}
$$


where

$$
\begin{aligned}
U(m) & =\left(1-c_{m}\right)^{2}\left(\psi\left(m, N_{t}\right)-N_{t}+1\right)+c_{m}^{2}\left(N_{t} \psi\left(m, N_{t}\right)\right. \\
& \left.-\frac{N_{t}}{L^{2}} \varphi^{2}(M)+1\right)
\end{aligned}
$$

and

$$
\begin{aligned}
V(m) & =\frac{2}{L}\left(1-c_{m}\right) c_{m}\left(N_{t}-N_{t} \psi\left(m, N_{t}\right)+1\right) \\
& \times \varphi(M) \cos \left(\frac{\pi M(L-1)}{N}\right) .
\end{aligned}
$$

Applying RVQ to quantize $K$ beamforming vectors with $B$ feedback bits and interpolating the rest with linear weight $c_{m}$ and phase rotation $\theta_{m}$, the associated sum capacity is given by

$$
C=\sum_{k} \sum_{m} E \log \left(1+\rho\left|\boldsymbol{H}_{k M+m}^{\dagger} \boldsymbol{v}_{k M+m}\right|^{2}\right) .
$$

\section{B. MIMO Channel}

For MIMO channel, $\boldsymbol{H}_{n}$ is an $N_{r} \times N_{t}$ matrix and the optimal beamforming vector for the $n$th subcarrier $\boldsymbol{v}_{n}^{\text {opt }}$ is the eigenvector of channel covariance $\boldsymbol{H}_{n}^{\dagger} \boldsymbol{H}_{n}$ corresponding to the maximum eigenvalue. Evaluating $E\left|\left(\boldsymbol{v}_{n}^{\text {opt }}\right)^{\dagger} \boldsymbol{v}_{n+q}^{\text {opt }}\right|^{2}$ is not tractable since a distribution function of the eigenvector is extremely complex. However, we observe from numerical examples that the correlation between channel matrices at different subcarriers can be a good approximation as follows

$$
E\left|\left(\boldsymbol{v}_{n}^{\mathrm{opt}}\right)^{\dagger} \boldsymbol{v}_{n+q}^{\mathrm{opt}}\right|^{2} \approx \frac{E\left|\operatorname{vec}\left(\boldsymbol{H}_{n}\right)^{\dagger} \operatorname{vec}\left(\boldsymbol{H}_{n+q}\right)\right|^{2}}{E\left[\left\|\operatorname{vec}\left(\boldsymbol{H}_{n}\right)\right\|^{2}\left\|\operatorname{vec}\left(\boldsymbol{H}_{n+q}\right)\right\|^{2}\right]}
$$

where $\operatorname{vec}\left(\boldsymbol{H}_{n}\right)$ converts the $N_{r} \times N_{t}$ matrix $\boldsymbol{H}_{n}$ into an $N_{t} N_{r} \times 1$ vector by stacking columns of the matrix $\boldsymbol{H}_{n}$ on top of one another. Since all transmit-receive antenna pairs are independent, evaluating the right-hand side of (17) is similar to that for MISO channel. Thus, it is straightforward to show that

$$
\frac{E\left|\operatorname{vec}\left(\boldsymbol{H}_{n}\right)^{\dagger} \operatorname{vec}\left(\boldsymbol{H}_{n+q}\right)\right|^{2}}{E\left[\left\|\operatorname{vec}\left(\boldsymbol{H}_{n}\right)\right\|^{2}\left\|\operatorname{vec}\left(\boldsymbol{H}_{n+q}\right)\right\|^{2}\right]}=\psi\left(m, N_{t} N_{r}\right)
$$

where $\psi(\cdot, \cdot)$ is defined in (12). Replacing $\psi\left(m, N_{t}\right)$ with $\psi\left(m, N_{t} N_{r}\right)$ in (13)-(15), the phase rotation $\theta_{m}$ for MIMO channel is obtained.

The associated sum capacity for MIMO channel with this proposed beamforming quantization and interpolation scheme is also given by (16).

\section{Quantizing Channel Impulse Response}

When available feedback rate is sufficiently high, quantizing channel impulse response directly can give a good performance [9]. Here we propose to quantize all channel taps of all transmit-receive antenna pairs with a scalar uniform quantizer. A uniform quantizer is simple and performs close to the optimal quantizer when the number of quantization bits is high. Real and imaginary parts of all channel taps are quantized independently with the same number of bits, which is $\frac{B}{2 N_{t} N_{r} L}$. Thus, the quantized $l$ th channel tap for the $\left(n_{r}, n_{t}\right)$ antenna pair is given by

$$
\begin{aligned}
\hat{h}_{n_{r}, n_{t}}^{(l)} & =\hat{h}_{n_{r}, n_{t}, r}^{(l)}+j \hat{h}_{n_{r}, n_{t}, i}^{(l)} \\
& =Q\left(h_{n_{r}, n_{t}, r}^{(l)}\right)+j Q\left(h_{n_{r}, n_{t}, i}^{(l)}\right)
\end{aligned}
$$

where $h_{n_{r}, n_{t}, r}^{(l)}$ and $h_{n_{r}, n_{t}, i}^{(l)}$ are real and imaginary parts of $h_{n_{r}, n_{t}}^{(l)}, Q(\cdot)$ is the uniform scalar quantizer with $2^{\frac{B}{2 N_{t} N_{r} L}}$ steps, while variables with hats denote outputs of the quantizer. Here we select a step size of the quantizer by the existing rule of thumb for Gaussian input (cf. [10, p. 125])

$$
\Delta=\frac{4 E\left[\left(h_{n_{r}, n_{t}, r}^{(l)}\right)^{2}\right]}{2^{\frac{B}{2 N_{t} N_{r} L}}}=\frac{1}{\sqrt{L}} 2^{\frac{3}{2}-\frac{B}{2 N_{t} N_{r} L}},
$$

which changes with the variance of channel tap and the number of quantization bits. Then, the transmitter computes a DFT of the quantized channel impulse response to obtain an approximate frequency response as follows

$$
\hat{H}_{n_{r}, n_{t}}(n)=\hat{\boldsymbol{h}}_{n_{r}, n_{t}}^{T} \boldsymbol{D}_{n}=\sum_{l=0}^{L-1} \hat{h}_{n_{r}, n_{t}}^{(l)} \mathrm{e}^{-\frac{j 2 \pi l n}{N}},
$$

which is the $\left(n_{r}, n_{t}\right)$ entry of the quantized $N_{r} \times N_{t}$ channel matrix for the $n$th subcarrier denoted by $\hat{\boldsymbol{H}}_{n}=\left[\hat{H}_{n_{r}, n_{t}}(n)\right]$.

Based on $\hat{\boldsymbol{H}}_{n}$, the transmitter is able to compute the optimal transmit beamforming vector. For MISO channel $\left(N_{r}=1\right)$, the optimal transmit beamforming vector based on quantized channel is given by

$$
\hat{\boldsymbol{v}}_{n}^{\mathrm{opt}}=\hat{\boldsymbol{H}}_{n}^{\dagger} /\left\|\hat{\boldsymbol{H}}_{n}\right\|
$$

and the corresponding sum rate over all subcarriers is given by

$$
\begin{aligned}
C & =\sum_{n=0}^{N-1} E\left[\log \left(1+\rho\left|\boldsymbol{H}_{n} \hat{\boldsymbol{v}}_{n}^{\mathrm{opt}}\right|^{2}\right)\right] \\
& =N E\left[\log \left(1+\rho \frac{\left|\boldsymbol{H}_{n} \hat{\boldsymbol{H}}_{n}^{\dagger}\right|^{2}}{\left\|\hat{\boldsymbol{H}}_{n}\right\|^{2}}\right)\right]
\end{aligned}
$$

where we substitute (23) into (24) and use the fact that distribution of the received power $\left|\boldsymbol{H}_{n} \hat{\boldsymbol{v}}_{n}^{\text {opt }}\right|^{2}$ for each subcarrier is identical. Furthermore, we apply Jensen's inequality and approximate a expectation of quotient by a quotient of the two expectations. Consequently, the approximate upper bound of the sum capacity is given by

$$
C \lesssim N \log \left(1+\rho \frac{E\left|\boldsymbol{H}_{n} \hat{\boldsymbol{H}}_{n}^{\dagger}\right|^{2}}{E\left\|\hat{\boldsymbol{H}}_{n}\right\|^{2}}\right) .
$$

Applying the fact that real and imaginary parts of channel taps are independent Gaussian distributed with zero mean, variance $\frac{1}{2 L}$, and a probability density function for $h_{n_{r}, n_{t}, r}^{(l)}$ denoted by $f_{h_{r}}(\cdot)$, we can show that

$$
E\left\|\hat{\boldsymbol{H}}_{n}\right\|^{2}=N_{t}\left(1-2 L E\left[\left(\hat{h}_{r}-h_{r}\right)^{2}\right]\right)
$$


and

$$
\begin{aligned}
E\left|\boldsymbol{H}_{n} \hat{\boldsymbol{H}}_{n}^{\dagger}\right|^{2}=N_{t}( & +\frac{1}{L}-(2 L-1) E\left[\left(\hat{h}_{r}-h_{r}\right)^{2}\right] \\
& \left.+2 L E\left[\hat{h}_{r}^{2} h_{r}^{2}\right]+4 L\left(N_{t} L-1\right) E^{2}\left[\hat{h}_{r} h_{r}\right]\right)
\end{aligned}
$$

where the mean squared error is given by

$$
E\left[\left(\hat{h}_{r}-h_{r}\right)^{2}\right]=\int(Q(x)-x)^{2} f_{h_{r}}(x) \mathrm{d} x
$$

and the correlation and its second moment are given by

$$
\begin{gathered}
E\left[\hat{h}_{r} h_{r}\right]=\int x Q(x) f_{h_{r}}(x) \mathrm{d} x \\
E\left[\hat{h}_{r}^{2} h_{r}^{2}\right]=\int x^{2} Q^{2}(x) f_{h_{r}}(x) \mathrm{d} x .
\end{gathered}
$$

We remark that without loss of clarity, we have dropped indices $n_{t}, n_{r}$, and $l$ from random variable $h_{n_{r}, n_{t}, r}^{(l)}$.

Each term in (29)-(31) can be computed numerically. However, to obtain some insight on how the sum capacity depends on a feedback rate and other channel parameters, we approximate each term in a high feedback-rate regime. It was shown that for large $B$ [11],

$$
E\left[\left(\hat{h}_{r}-h_{r}\right)^{2}\right] \approx \frac{\Delta^{2}}{12}=\frac{2}{3 L} 2^{-\frac{B}{N_{t} L}} .
$$

Applying property of the optimum quantizer [12], we obtain

$$
E\left[\hat{h}_{r} h_{r}\right] \approx \frac{1}{2 L}-E\left[\left(\hat{h}_{r}-h_{r}\right)^{2}\right] .
$$

As $B \rightarrow \infty, \hat{h}_{r} \rightarrow h_{r}$. Hence,

$$
\lim _{B \rightarrow \infty} E\left[\hat{h}_{r}^{2} h_{r}^{2}\right]=\frac{3}{4 L^{2}}
$$

Substituting (32)-(34) into (27) and (28), we obtain the approximate upper bound for a sum capacity for MISO channel with large $B$ as follows

$$
C \lesssim N \log \left(1+\rho\left(1-\frac{1}{L}+\left(N_{t} L-1\right) \Xi_{B}+\frac{3}{4 L^{2} \Xi_{B}}\right)\right)
$$

where $\Xi_{B}=\frac{1}{L}-\frac{4}{3 L} 2^{-\frac{B}{N_{t} L}}$.

For MIMO channel, the optimal transmit beamformer $\hat{\boldsymbol{v}}_{n}^{\mathrm{opt}}$ is the eigenvector of $\hat{\boldsymbol{H}}_{n} \hat{\boldsymbol{H}}_{n}^{\dagger}$. Deriving a similar sum capacity requires $E\left|\boldsymbol{H}_{n+q} \hat{\boldsymbol{v}}_{n}^{\mathrm{opt}}\right|^{2}$, which is intractable. Thus, an analytical approximation of the sum capacity for MIMO channel remains an open problem.

\section{Numerical Results}

In Fig. 1, we show averaged capacity per subcarrier for MISO-OFDM channel with the two proposed feedback methods and the interpolation method by [4]. When the number of feedback bits $B$ or the number of transmit antennas $N_{t}$ increase, the capacity increases as expected. For a low feedback rate, our beamforming interpolation method performs better than that by [4]. We also note that the method by [4] requires some minimum number of feedback bits, which is used to quantize the phase-rotation parameter $\theta_{m}$. For both interpolation schemes, beamformers at every $M=8$ subcarriers are quantized while the rest are interpolated.

For a high feedback rate, quantizing channel taps directly performs comparably to the interpolation method by [4]. We observe that for $N_{t}=3$, switching from beamforming interpolation to quantization of channel taps should occur when $B=50$, and for $N_{t}=5$, it should occur when $B=80$. We also note that for $N_{t}=3$, feeding back 100 bits can potentially increase capacity by $60 \%$ compared to the zerofeedback capacity.

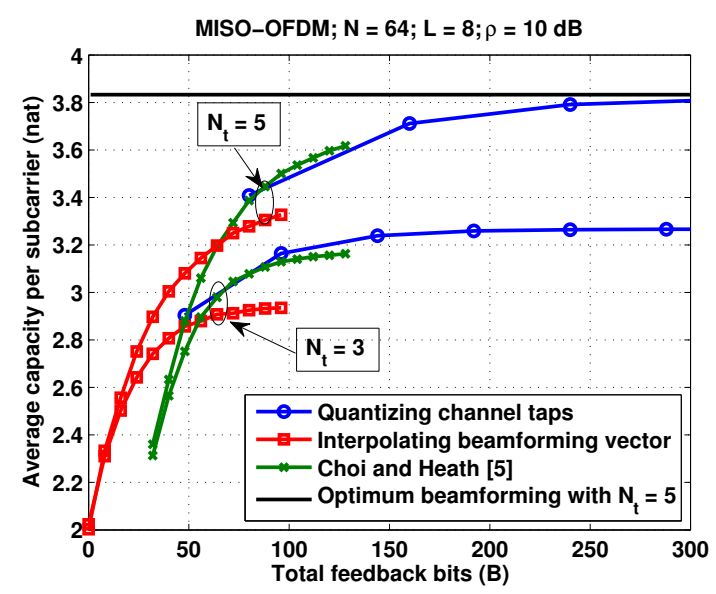

Fig. 1. Average capacity for MISO-OFDM with different feedback methods are shown with $B$ and $N_{t}$ for $N=64, L=8, M=8$, and $\rho=10 \mathrm{~dB}$.

For a $3 \times 2$ MIMO channel, the average capacity per subcarrier is shown in Fig. 2 with total feedback bits $B$ and different number of channel taps for $N=64, M=8$, and $\rho=10 \mathrm{~dB}$. We remark that for a very low feedback rate, the proposed interpolation method outperforms the method by [4]. For MIMO channel in general, quantizing channel taps requires much larger $B$ than the interpolation method does. For example, allocating 4 quantization bits for each complex channel tap leads to the total feedback bits $B=4 N_{t} N_{r} L$, which can be unreasonably large. For this numerical example, it turns out that quantizing channel taps needs more than 140 bits and hence, we omit its performance plot.

In Fig. 3, we compare a capacity per subcarrier of $3 \times 1$ channel obtained from simulation and the approximate upper bound (35) for a direct quantization of channel taps. A number of channel taps $L$ varies between 4 to 16 . From the figure, the approximate upper bound exhibits the same performance trend as simulation results and the gap between the two is about $12 \%$. Although the approximation is derived for a large feedback rate, it seems to predict the simulation result even with relatively small $B$ well. In addition, we observe from simulation results that approximately 3 bits per real coefficient is needed to achieve close to the capacity maximum. While the number of fading paths $L$ increases, $B$ also increases to achieve close to the capacity maximum.

The last figure shows capacity per subcarrier for a $3 \times 1$ OFDM channel with beamforming interpolation for severely 


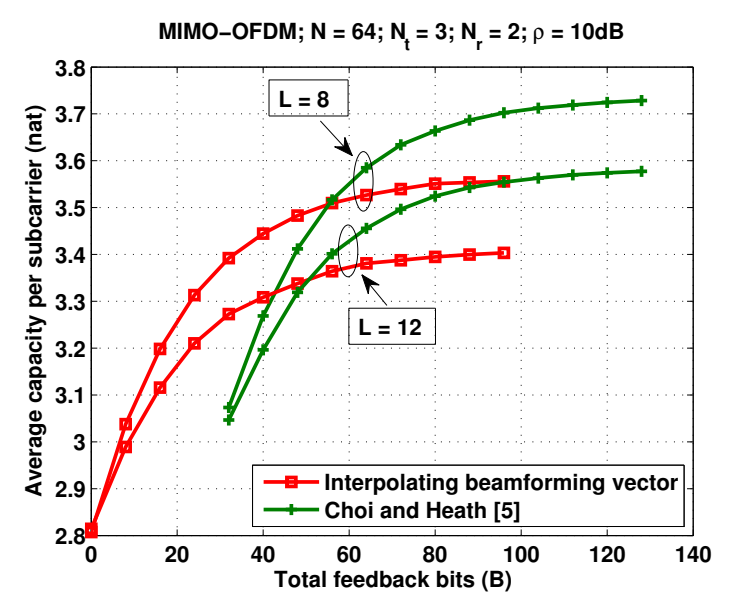

Fig. 2. Shown is a capacity per subcarrier of a $3 \times 2$ OFDM channel with beamforming interpolation for $N=64, M=8$, and $\rho=10 \mathrm{~dB}$.

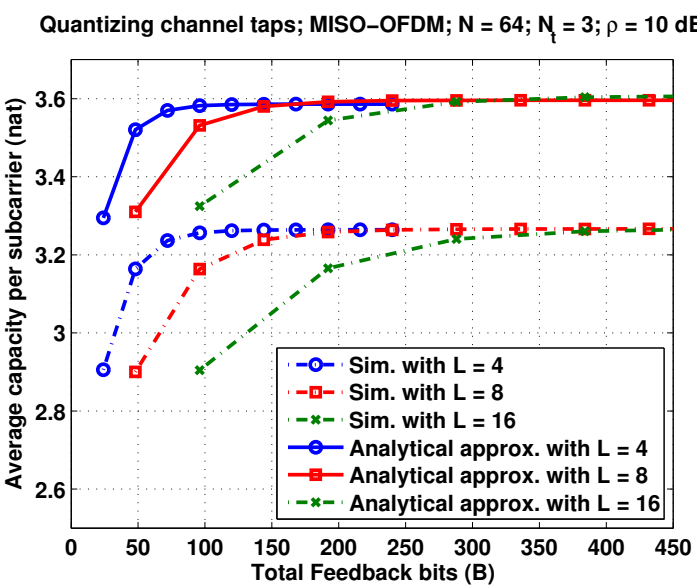

Fig. 3. A comparison between capacity obtained from simulation and the analytical upper bound for the channel-tap quantization is shown with $N=$ $64, N_{t}=3$, and $\rho=10 \mathrm{~dB}$.

limited feedback rate, $B=32$. Different plots correspond to different $L$ and are shown with $M$, which denotes the number of subcarriers between adjacent quantized beamformers. For small $M$, more beamforming vectors are quantized and fed back from the receiver, but with smaller number of feedback bits per vector. For large $M$, the opposite is true. Thus, there exists the optimal $M$ that maximizes the capacity. For a flat fading channel $(L=1)$, frequency response is constant across subcarriers and there is only a need for one quantized beamforming vector with all available feedback bits. Thus, the optimal $M=N$. For larger $L$, channel becomes more frequency-selective and thus, the optimal $M$ is smaller. Based on this numerical example, we should operate with $M=16$ when $L=4$ and $B=32$.

\section{CONCLUSIONS}

In this paper, we have proposed a beamforming interpolation, which can perform well with very limited feedback, and quantization of channel impulse response, which needs high feedback rate to perform well. Thus, switching between

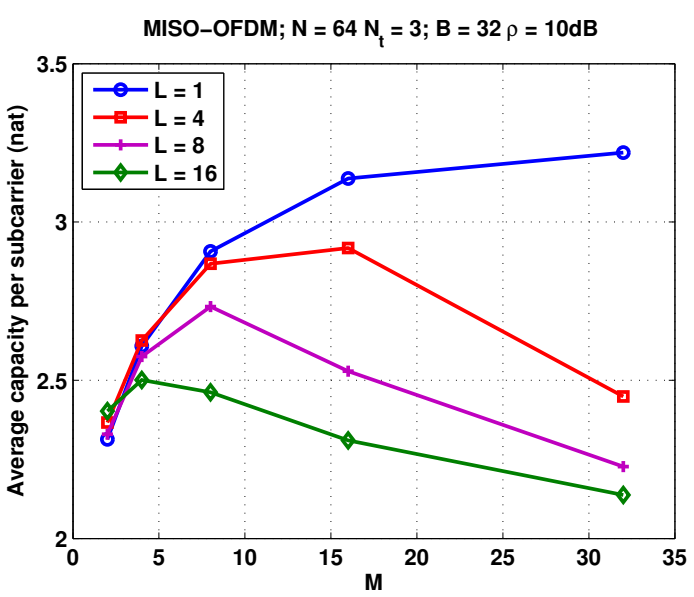

Fig. 4. Average capacity of a $3 \times 1$ OFDM channel with beamforming interpolation is shown with $M$ for different $L, N=64, B=32$, and $\rho=10 \mathrm{~dB}$

the two methods for different feedback rate is recommended. We have also analyzed the capacity with direct quantization of channel taps, which depends on feedback rate and the number of antennas and channel taps. Our future work includes analyzing the capacity with the interpolation method and the optimal subcarrier interval for quantizing beamformers.

\section{REFERENCES}

[1] T. K. Y. Lo, "Maximum ratio transmission," IEEE Trans. Commun., vol. 47, no. 10, pp. 1458-1461, Oct. 1999.

[2] W. Santipach and M. L. Honig, "Capacity of a multiple-antenna fading channel with a quantized precoding matrix," IEEE Trans. Inf. Theory, vol. 55, no. 3, pp. 1218-1234, Mar. 2009.

[3] D. J. Love, R. W. Heath, Jr, V. K. N. Lau, D. Gesbert, B. D. Rao, and M. Andrews, "An overview of limited feedback in wireless communication systems," IEEE J. Sel. Areas Commun., vol. 26, no. 8, pp. 1341-1365, Oct. 2008.

[4] J. Choi and R. W. Heath, Jr., "Interpolation based transmit beamforming for MIMO-OFDM with limited feedback," IEEE Trans. Signal Process., vol. 53, no. 11, pp. 4125 - 4135, Nov. 2005.

[5] K. Mamat and W. Santipach, "Subcarrier clustering for MISO-OFDM channels with quantized beamforming," in Proc. ECTI-CON, Huahin, Thailand, May 2012, pp. 1-4.

[6] C. He, P. Zhu, B. Sheng, and X. You, "Two novel interpolation algorithms for MIMO-OFDM systems with limited feedback," in Proc. IEEE Veh. Technol. Conf. (VTC Fall), San Francisco, CA, Sep. 2011, pp. $1-5$.

[7] Q. Huang, M. Ghogho, Y. Li, D. Ma, and J. Wei, "Transmit beamforming for MISO frequency-selective channels with per-antenna power constraint and limited-rate feedback," IEEE Trans. Veh. Technol., vol. 60, no. 8, pp. 3726-3735, Oct. 2011.

[8] C. K. Au-Yeung and D. J. Love, "On the performance of random vector quantization limited feedback beamforming in a MISO system," IEEE Trans. Wireless Commun., vol. 6, no. 2, pp. 458-462, Feb. 2007.

[9] D. J. Love, R. W. Heath, Jr., W. Santipach, and M. L. Honig, "What is the value of limited feedback for MIMO channels?" IEEE Commun. Mag., vol. 42, no. 10, pp. 54-59, Oct. 2004.

[10] N. S. Jayant and P. Noll, Digital Coding of Waveforms: Principles and Applications to Speech and Video. Englewood Cliffs, NJ: Prentice-Hall, 1984.

[11] D. Hui and D. L. Neuhoff, "Asymptotic analysis of optimal fixed-rate uniform scalar quantization," IEEE Trans. Inf. Theory, vol. 47, no. 3, pp. 957-977, Mar. 2001.

[12] J. Bucklew and N. Gallagher, Jr., "A note on optimal quantization," IEEE Trans. Inf. Theory, vol. 25, no. 3, pp. 365-366, May 1979. 